\title{
Coexistent Sarcoidosis and Tuberculosis: A Case Report
}

\author{
Cristiano Carbonelli ${ }^{a, b}$ Ernesto Giuffreda ${ }^{a}$ Antonio Palmiotti ${ }^{a}$ \\ Domenico Loizzic ${ }^{c}$ Filippo Lococo $^{d}$ Elisiana Carpagnano ${ }^{a}$ Donato Lacedonia ${ }^{a}$ \\ Francesco Sollitto $^{c}$ Maria Pia Foschino ${ }^{a}$ \\ ${ }^{a}$ Department of Medical and Surgical Sciences, Institute of Respiratory Diseases, Azienda Ospedaliero Universitaria

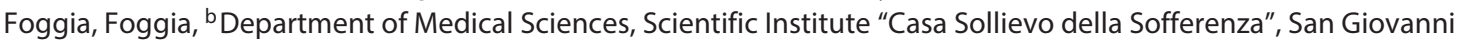 \\ Rotondo, 'Department of Thoracic Surgery, University of Foggia, Foggia, and ' Unit of Thoracic Surgery, Arcispedale \\ Santa Maria Nuova-IRCCS, Reggio Emilia, Italy
}

\section{Case Presentation}

A 45-year-old black male with a medical history of chronic hepatitis $\mathrm{C}$ virus treated with pegylated interferon and ribavirin until 4 months before hospitalization was admitted for a bacterial pneumonitis. The examinations revealed neck, mediastinal, and abdominal adenopathies. Histologically, a Zielh-Neelsen-negative necrotizing granulomatous reaction was documented in a later cervical lymph node analysis. The Mantoux test was negative, and bronchoalveolar lavage showed a lymphocytic alveolitis $60 \%$ of the total with a CD4/CD8 ratio of 4 . Angiotensin-converting enzyme was elevated, and there was no sign of uveal involvement or hypercalciuria. As the microbiological examinations for mycobacteria were repeatedly negative, even upon examination of a culture on respiratory and peripheral lymph node tissue, a provisional diagnosis of sarcoidosis was made, and the patient was discharged with a follow-up schedule without therapy apart from oral antiviral drugs (entecavir).

About 3 months later, the patient came to the Department of Infectious Disease of our hospital, complaining of high-grade septic fever and bilateral pleural effusions. A thoracic and abdominal CT confirmed few nodular elements in both lungs along with the confirmation of a pericardial and bilateral pleural effusion and multiple spleen nodules.

The analysis of the pleural fluid was unremarkable, apart from prevalence of lymphocytes upon cytological evaluation. As the patient was prostrated for the longlasting febrile manifestation, the introduction of steroid therapy led to the resolution of fever.

The patient's conditions ameliorated, and a chest Xray documented an improvement of the left pleural effusion. However, a worsening on the right side was seen with echo signs of multiloculation (Fig. 1).

What is your diagnosis?

\section{KARGER}

() 2017 S. Karger AG, Basel

E-Mail karger@karger.com

www.karger.com/res
Cristiano Carbonelli, MD

Department of Medical and Surgical Sciences, Institute of Respiratory Diseases Azienda Ospedaliero Universitaria Foggia, Viale degli Aviatori 1 IT-71100 Foggia (Italy)

E-Mail kkarbo@hotmail.com 


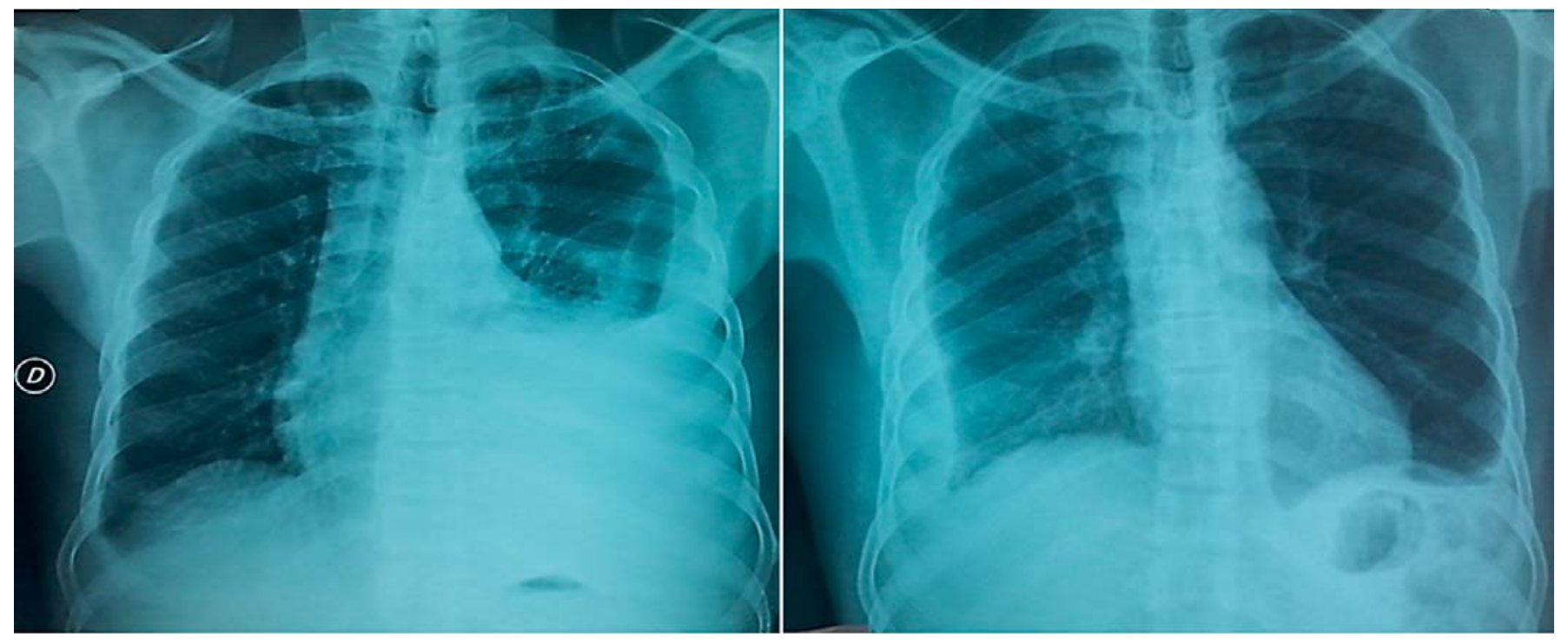

Fig. 1. Radiological and radiometabolic scenario. Chest X-rays showed the different evolution of pleural effusion with simultaneous improvement on the left side and worsening on the right side. 


\section{Diagnosis: Pleural Tuberculosis in the Framework of a Concomitant Systemic Sarcoid Manifestation}

A positron emission tomography/CT examination confirmed multiple areas of a high 18-fluorodeoxyglucose uptake at pleural surfaces, systemic lymphonodes, and the spleen (Fig. 2). The patient was referred to the interventional pulmonology service with the aim of performing a medical thoracoscopy for a definitive diagnosis. Geographic pleural thickening (Fig. 3a) with multiple multiloculations and a granulomatous necrotizing disease at the level of the pleural tissue were present (Fig. 3b). However, Zielh-Neelsen colorations were again negative, as were polymerase chain reaction analyses for tuberculosis (TB).

Quantiferon positivity, together with the endoscopic scenario and the histological pattern, motivated the introduction of antimicrobacterial treatment (isoniazid, rifampicin, ethambutol, and pyrazinamide) and the withdrawal of steroids. High-grade fever reappeared, and an increase of GGT, nausea, and abdominal pain were reported. Circulating CD4 were 173 cells $/ \mu \mathrm{L}$, and the HIV test was negative. Twenty days later, steroid reintroduction (methylprednisolone $1 \mathrm{mg} / \mathrm{kg}$ ) dramatically im- proved the fever and overall clinical scenario. Lymphocytic CD4 increased to 391 cells/ $\mu \mathrm{L} 2$ weeks after steroid introduction.

The positivity of cultures on pleural specimens for Mycobacterium tuberculosis was reported 45 days after thoracoscopy, leading to the confirmation of pleural TB in the framework of a concomitant systemic sarcoid manifestation.

\section{Discussion}

Nevertheless, the words "necrosis" and "pleural involvement" in relation with a granulomatous disease are usually interpreted as highly suggestive of TB. Differential diagnosis has to include well-described series of sarcoid pleural involvement. Pleural involvement is frequent in necrotizing sarcoid granulomatosis (NSG), and the treatment with steroids appears to improve the clinical findings [1].

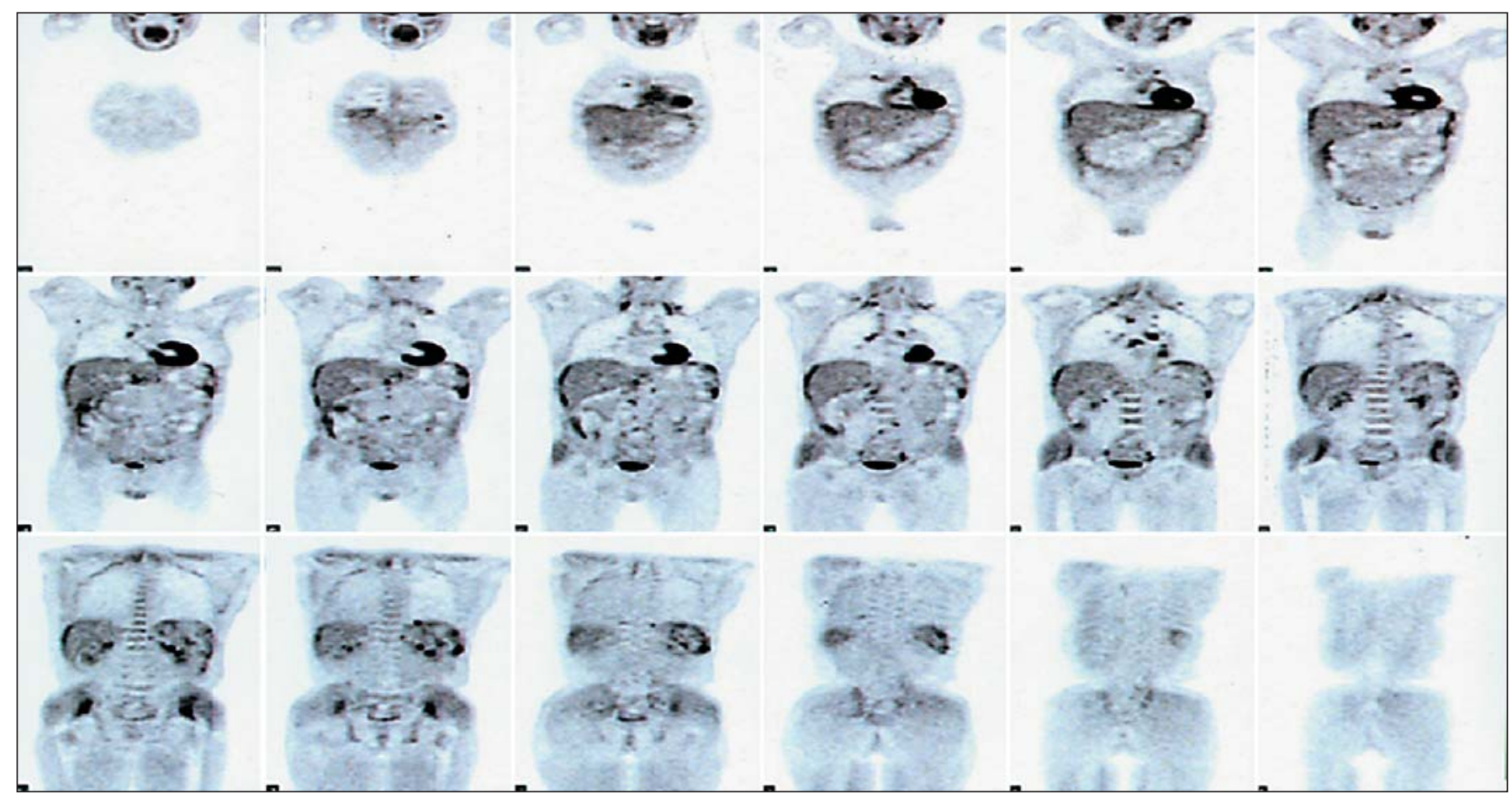

Fig. 2. Radiological and radiometabolic scenario. ${ }^{18} \mathrm{~F}-\mathrm{FDG}$ positron emission tomography/CT scan with multiple areas of mediastinal lymph nodes, pleural surfaces, and spleen, showing a high uptake of the radiotracer. 
Sarcoidosis represents a stereotyped, antigen-driven immune disorder with diverse etiologies [2]. A century of epidemiologic, molecular, and immunologic literature emphasizes the hypothesis about a causal role of mycobacteria antigens in some sarcoidosis cases $[3,4]$.

The unspecificity of the clinical findings in these cases is well recognized. The classic manifestations of sarcoidosis and TB have an overlapping range for which it is sometimes difficult to make a definitive diagnosis in the absence of a microbiological identification [5]. Hepatitis $\mathrm{C}$ virus may be a cofactor in the pathogenesis of sarcoidosis, and a close association between interferon therapy and sarcoidosis has been described [6].

Sarcoid granulomatosis and TB are sometimes entities difficult to separate, even on a pathological basis in the absence of a cultural microbiological confirmation. Indeed, there is a continuous spectrum of necrosis in sarcoidosis, ranging from minimal to massive grade. The socalled sarcoidosis with the NSG pattern requires a reasonable exclusion of an infectious etiology [7]. Failure to culture mycobacteria from sarcoidosis specimens may be secondary to difficulties in obtaining tissue from critically involved sites, microorganism peculiarity, bacteria burden, or inadequate characteristics of culture assays
[3]. Positron emission tomography imaging can be useful in order to guide the surgical biopsy in NSG [8].

Coexistence of TB and sarcoidosis is a rarely described [9] and likely a highly underrecognized entity [10]. Bronchoalveolar lavage does not accurately discriminate between sarcoidosis and other causes of lymphocytic alveolitis such as TB [11]. The case herein supports the hypothesis that systemic manifestation of sarcoidosis and its improvement with steroid treatment can occur coincidently with low-antigen, pleural TB.

Nuclear medicine techniques and thoracoscopy findings for tissue acquisition in the described diagnostic process must be considered to ascertain a mycobacterial infection as the causative agent of a concomitant sarcoid manifestation. The choice to continue both steroid and anti-TB therapy proved to be correct for the late evidence of TB mycobacterial growth only on pleural specimens. In most cases, the diagnosis of necrotizing sarcoidosis and TB with a low antigen load cannot be formulated, and physicians should still manage to protect pharmacologically the less likely clinical hypothesis and, at the same time, closely follow the clinical course and the outcome of the investigations carried out, avoiding irreparable damage in case of misdiagnosis.
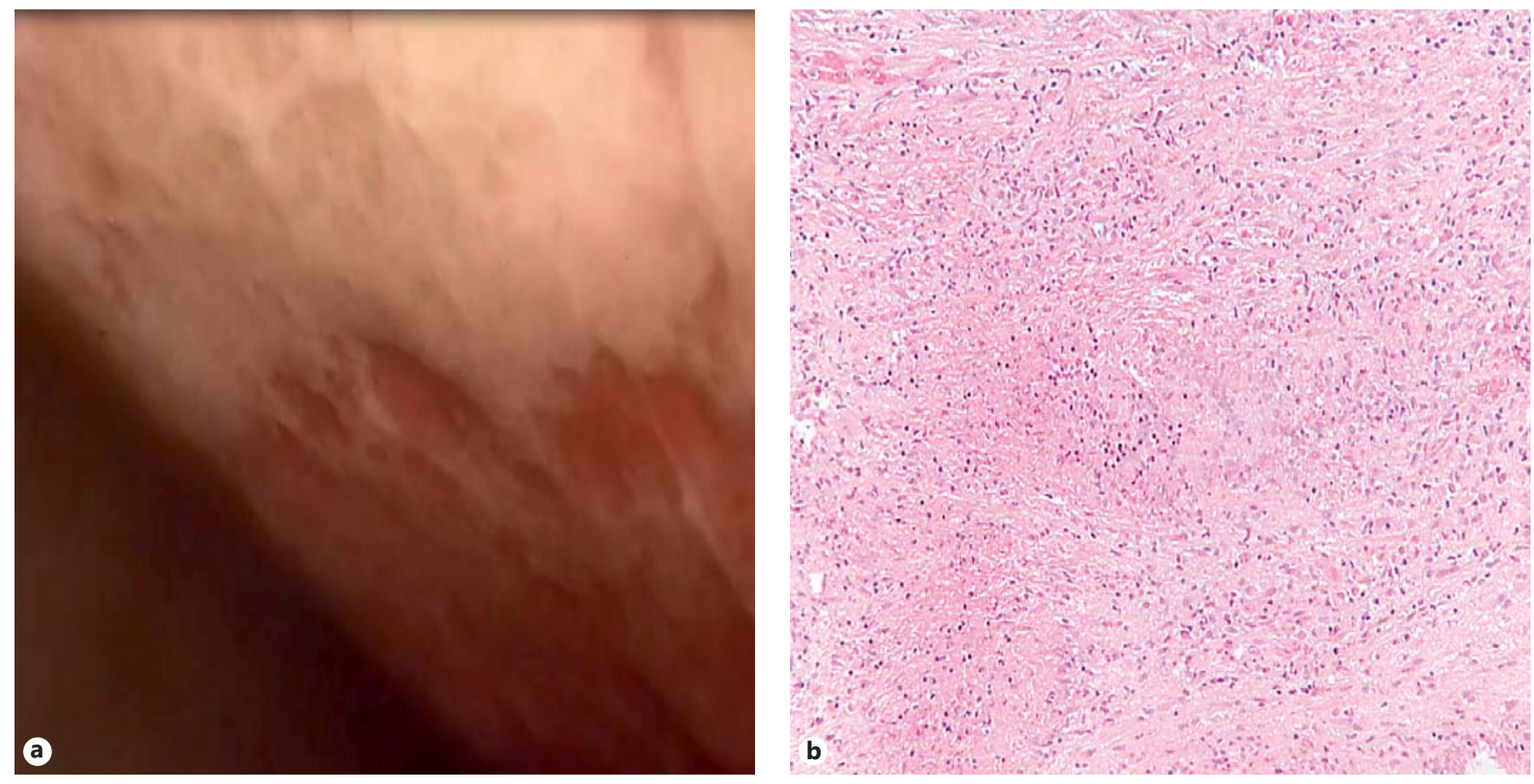

Fig. 3. Endoscopic view and pathological examination. Thoracoscopic view (a) of the right pleural cavity, showing disseminated fibrinous pleural thickenings with multiple multiloculations. Pathological examination of the pleural biopsy (b), revealing a granulomatous caseating necrotizing pattern. HE. $\times 200$. 


\section{Acknowledgement}

Thanks to the Pleural Hub group on Facebook for their assistance in the multidisciplinary discussion of the case.

Thanks to Ms. Anna T. Valencia, MPH, MBEMH, for language support.

\section{Statement of Ethics}

We obtained informed patient consent for the participation and publication of individual patient data.

\section{Disclosure Statement}

The authors declare that they have no competing interests to disclose.

\section{Author Contribution}

C. Carbonelli performed the interventional procedures, conceived the study, participated in its design and coordination, and drafted the manuscript.

E. Giuffreda performed the transthoracic echography and participated in the design of the study and in the drafting of the manuscript.

A. Palmiotti performed the transthoracic echography and participated in the design of the study.

D. Loizzi performed the interventional procedures and participated in the design of the study.

E. Carpagnano, D. Lacedonia, and F. Lococo participated in the design of the study and in the drafting of the manuscript.

F. Sollitto performed the interventional procedures and participated in the design of the study.

M.P. Foschino participated in the design of the study and its coordination as well as in the drafting of the manuscript.

All authors have read and approved the final manuscript.

\section{Keywords}

Pleural biopsy · Thoracoscopy · Sarcoidosis · Tuberculosis

Abstract

Necrotizing granulomatous diseases of the lungs are usually dependent on a narrow range of differential diagnoses. Tuberculosis (TB) is responsible for the largest number of cases, while necrotizing sarcoidosis is generally considered a rare and easily distinguishable disease substantially based on histological features. However, this entity has become a viable diagnosis in the absence of mycobacteria isolation or when a remarkable clinical improvement cannot be achieved with the combination of anti-TB drugs at full dosage. The classic manifestations of TB and sarcoidosis have an overlapping range for which it is sometimes difficult to make a clinical diagnosis. Furthermore, the role of mycobacteria as a trigger antigen capable of evoking the clinical expression of sarcoidosis is a hypothesis supported by evidence from some cases. We report a case of bilateral tuberculous pleurisy in a 45-year-old male native of a North-African region with an atypical severe multisystem disease characterized by a fever resistant to anti-TB therapy and respondent to corticosteroid treatment. The choice to continue both steroid and anti-TB therapy proved to be correct for the late evidence of TB mycobacterial growth only on pleural specimens. The case described is suggestive of a coexistent systemic sarcoid manifestation and low-antigen $\mathrm{TB}$, which is an underrecognized entity in the medical literature.

\section{References}

1 Chittock DR, Joseph MG, Paterson NA, McFadden RG: Necrotizing sarcoid granulomatosis with pleural involvement. Clinical and radiographic features. Chest 1994;106:672676.

2 Moller DR: Treatment of sarcoidosis - from a basic science point of view. J Intern Med 2003; 253:31-40.

3 Drake WP, Newman LS: Mycobacterial antigens may be important in sarcoidosis pathogenesis. Curr Opin Pulm Med 2006;12:359363.

4 Fité E, Fernández-Figueras MT, Prats R, Vaquero M, Morera J: High prevalence of Mycobacterium tuberculosis DNA in biopsies from sarcoidosis patients from Catalonia, Spain. Respiration 2006;73:20-26.
5 Agarwal R, Gupta D: Tuberculous sarcoidosis: is it a separate entity? Lung India 2009; 26:61-62.

6 Ramos-Casals M, Mañà J, Nardi N, BritoZeròn P, Xaubet A, Sãnchez-Tapias JM, Cervera R, Font J; HISPAMEC Study Group: Sarcoidosis in patients with chronic hepatitis $\mathrm{C}$ virus infection: analysis of 68 cases. Medicine (Baltimore) 2005;84:69-80.

7 Rosen Y: Four decades of necrotizing sarcoid granulomatosis. What do we know? Arch Pathol Lab Med 2015;139:252-262.

8 Arfi J, Kerrou K, Traore S, Huchet V, Bolly A, Antoine M, Delaunois L, Vander Borght T, Talbot JN: F-18 FDG PET/CT findings in pulmonary necrotizing sarcoid granulomatosis. Clin Nucl Med 2010;35:697-700.
9 Wong CF, Yew WW, Wong PC, Lee J: A case of concomitant tuberculosis and sarcoidosis with mycobacterial DNA present in the sarcoid lesion. Chest 1998;114:626-629.

10 Lin JY, Sheu SJ: Ocular sarcoidosis and tuberculous lymphadenopathy: coincidence or real association. J Ophthal Inflamm Infect 2011; 137-140.

11 Bretagne L, Diatta I-D, Faouzi M, Nobile A, Bongiovanni M, Nicod LP, Lazor R: Diagnostic value of the CD103+CD4+/CD4+ ratio to differentiate sarcoidosis from other causes of lymphocytic alveolitis. Respiration 2016;91: 486-496. 